\title{
openheart Evaluation of intermediate coronary stenoses in acute coronary syndromes using pressure guidewire
}

\author{
Giampaolo Niccoli, ${ }^{1}$ Ciro Indolfi, ${ }^{2}$ Justin E Davies ${ }^{3}$
}

To cite: Niccoli G, Indolfi C, Davies JE. Evaluation of intermediate coronary stenoses in acute coronary syndromes using pressure guidewire. Open Heart 2017;4:e000431. doi:10.1136/ openhrt-2016-000431

Received 9 March 2016 Revised 15 August 2016 Accepted 30 August 2016

\section{CrossMark}

${ }^{1}$ Department of Cardiovascular Medicine, Institute of Cardiology, Catholic University of the Sacred Heart, Rome, Italy

${ }^{2}$ Division of Cardiology, Department of Medical and Surgical Sciences \& URT CNR, Magna Graecia University, Catanzaro, Italy

${ }^{3}$ National Heart and Lung Institute, International Centre for Circulatory Health, Imperial College London and Imperial College Healthcare NHS Trust, London, UK

Correspondence to Dr Giampaolo Niccoli; gniccoli73@hotmail.it

\section{ABSTRACT}

Fractional flow reserve (FFR) is increasingly used to guide myocardial revascularisation. However, supporting evidence regarding its use originates from studies that have enrolled mainly patients with stable angina, while patients with acute coronary syndromes (ACS) have not been included. Notably, multifactorial microvascular dysfunction and an increased sympathetic tone in patients with ACS may lead to blunted response to adenosine and false-negative results of FFR due to submaximal hyperaemia. This may raise the possibility of deferring treatment of stenosis that instead would have needed dilatation, thus leaving a residual risk of preventable cardiac events. In this literature review, we aim at summarising laboratory and clinical investigations concerning the use of FFR in culprit and non-culprit lesions in ACS. Furthermore, we will report recent data on instantaneous wave-free ratio, an adenosine-free index of functional stenosis severity, in stable coronary artery disease and in patients with ACS.

\section{INTRODUCTION}

Invasive functional evaluation of intermediate coronary stenosis, stenosis determining a luminal diameter narrowing of $\geq 40 \%$ but $\leq 70 \%$, is increasingly used for decision making in patients with coronary artery disease (CAD) ${ }^{1}$ Fractional flow reserve (FFR) has been reported to identify ischaemia-producing lesions with high accuracy. ${ }^{1}$ The latest (2014) European Society of Cardiology (ESC) guidelines on myocardial revascularisation suggest that, when non-invasive stress imaging is contraindicated, non-diagnostic or unavailable, the measurement of FFR is recommended during diagnostic coronary angiography. ${ }^{2}$ Deferral of percutaneous coronary intervention (PCI) or coronary artery bypass graft in patients with FFR $>0.80$ appears safe. ${ }^{2}$ However, this evidence originates from studies that have enrolled mainly patients with stable angina $(\mathrm{SA})^{34}$ and data about the role of FFR guidance in patients with acute coronary syndromes (ACS) are limited.

Of note, an essential prerequisite for FFR calculation is the achievement of "maximal" hyperaemia, usually accomplished by adenosine administration. ${ }^{1}$ Patients with ACS may however have mechanisms leading to blunted response to adenosine and submaximal hyperaemia (figure 1). Thus, normal FFR value $(>0.80)$ may indeed be falsely negative due to incomplete hyperaemia. This phenomenon may be true for both culprit and non-culprit lesions in ACS and is dependent on raised microvascular resistance and reduced coronary flow reserve (CFR) as recently summarised by Echavarría-Pinto et $a .^{5}$ Importantly, the possibility of false-negative results of FFR is increased around the grey zone of FFR and values of FFR between 0.81 and 0.85 should be managed cautiously as a residual risk of events may be present. ${ }^{6}$

The goal of this literature review is to summarise laboratory and recent clinical investigations concerning the use of FFR in culprit and non-culprit lesions in ACS mainly because these studies carry important implications both for treatment and prognosis. Particular emphasis will be placed on the potential current data on the use, and potential limitations of FFR in routine clinical practice for assessment of patients with ACS. Finally, recent data on instantaneous wavefree ratio (iFR), an adenosine-free index of functional stenosis severity, will be reviewed in detail, particularly for the possibility to assess the functional relevance of coronary stenosis without the need of adenosine administration.

\section{CURRENT CLINICAL EVIDENCE ON FFR IN ACS Culprit lesions in ST-elevation myocardial infarction}

Although the feasibility of making measurements was recently evaluated, no data have been published to show an improvement of clinical outcome when using FFR of the culprit lesion in the acute phase of the ST-elevation myocardial infarction (STEMI), thus currently it is not indicated. ${ }^{7}$ Of note, the percentage of intermediate coronary stenosis 


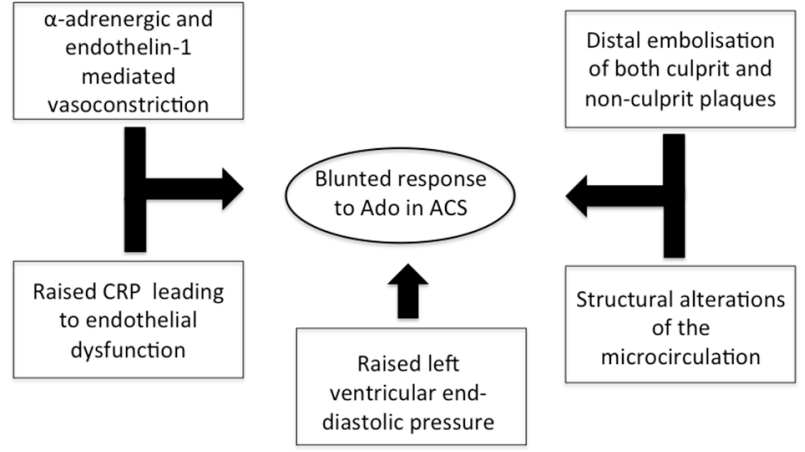

Figure 1 Mechanisms leading to blunted response to Ado and submaximal hyperaemia in patients with ACS. ACS, acute coronary syndrome; Ado, adenosine; CRP, C-reactive protein.

in culprit vessels of patients with STEMI accounts for less than $10 \% .{ }^{8}$ FFR could be indicated later after a successful thrombolysis. However, it is still unknown how long it takes for microvascular stunning following an acute coronary episode to resolve. In fact, the time to recovery of the microvasculature is variable, depending on the size of infarction, and the occurrence of microvascular obstruction (MVO). The longevity of MVO can be as short as days, and as long as a week, or more. ${ }^{79}$ The variable degree of reversible microvascular stunning reduces the maximal achievable flow resulting in smaller pressure gradients and their associated higher FFR values. ${ }^{1-7}$ 9-11 This phenomenon increases the chance of a false-negative FFR measurement. However, due to the phenomena described above, any physiological index may be questionable for the evaluation of the culprit vessel. Conversely, low FFR values should be considered reliable. Small studies have suggested that FFR assessment is more reliable between 4 and 6 days following the index event, ${ }^{12-14}$ as soon as the microcirculation can dilate sufficiently to enable maximal hyperaemia.

\section{Non-culprit lesions in STEMI}

A substantial percentage $(30 \%-50 \%)$ of patients with STEMI have multivessel coronary disease (MVD). These patients are a subgroup at high risk for major adverse cardiac events (MACE) in the first year after primary PCI for STEMI. ${ }^{10}$

Since intermediate stenosis rate in non-culprit vessels of STEMI accounts for about $70 \%$, the evaluation of non-culprit lesions represents an important clinical issue. ${ }^{15}$ Of note, in this setting of patients, a coronary and peripheral vasoconstriction due to alpha-adrenergic stimulation may occur and even angioplasty could induce a subsequent vasoconstriction. ${ }^{16}$ Therefore, how and when to interrogate a non-culprit intermediate stenosis is still unknown. The first question is the reliability of FFR of non-culprit lesions in the acute phase of STEMI. Ntalianis et alreported the reproducibility of FFR measurements in 101 patients undergoing PCI for acute myocardial infarction (MI) (75 STEMI and 26 non-ST-elevation myocardial infarction (NSTEMI)). ${ }^{15}$ FFR measurements were originally made in the non-culprit lesions at the time of culprit vessel PCI and then repeated $35 \pm 4$ days later. In a subgroup of 14 patients, the index of microvascular resistance (IMR) was also measured at the time of culprit vessel PCI and repeated $35 \pm 4$ days later. ${ }^{15}$ Although the mean value of FFR of non-culprit coronary artery stenoses during the acute phase and at follow-up was not statistically different, some FFR values crossed important clinical cut-off points (around $10 \%$ of patients). Of note, the recent DANAMI3-PRIMULTI study showed that the complete revascularisation using FFR-guided PCI in patients with STEMI and MVD reduced the risk of future events at a median follow-up of 27 months, compared with no further invasive intervention after primary PCI. ${ }^{17}$ However, many issues remain open. First of all, since the FFR measurements were done at a median of 2 days after the initial PCI procedure, the study might be affected by disturbances in microvascular function and oedema in the area. Second, the rate of events at follow-up in deferred lesions by FFR is not reported, thus not allowing again the assessment of residual risk carried by FFR guidance in ACS.

\section{FFR in NSTEMI}

Two clinical studies have assessed the impact of the use of FFR in NSTEMI. The first clinical study was FAME which included 328 patients with ACS out of a total of 1005 patients with MVD. ${ }^{18}$ Patients were enrolled with unstable angina (UA) or NSTEMI with positive troponin but total creatine kinase $<1000 \mathrm{U} / \mathrm{L}$. FFR guidance was associated with a $5.1 \%$ absolute and a $3.7 \%$ relative risk reduction after 2 years (combined end point of death, MI or revascularisation), similar to that obtained in the remainder of the patients with stable CAD. This ACS substudy was a subgroup analysis which was not powered to detect superiority of FFR in non-culprit ACS. Furthermore, theoretical limitation surrounds the time of FFR interrogation (within 5 days of admission but the exact time is not reported) that may affect FFR results due to the evolving status of the coronary microcirculation. Finally, neither the actual lesion assessed (culprit vs non-culprit) nor the event rate in deferred lesions by FFR in FAME is reported.

The second clinical study, FAMOUS-NSTEMI trial, ${ }^{19}$ randomised 350 patients with NSTEMI with at least one coronary stenosis of $\geq 30 \%$ severity by visual assessment of either FFR-guided versus angiography-only approach. The reported percentage of intermediate lesions was $25 \%$. The study showed an increased proportion of patients treated initially by medical therapy in the FFR-guided group when compared with the angio-guided group ( $22.7 \%$ vs $13.2 \%$ ), this is despite 12 MACE in both groups being similar (figure 2). The median time from the index episode of myocardial ischaemia to angiography was 3 days. A trend for more periprocedural MI in angio-guided procedure and for more MACE in the FFR guided-procedure was observed. Interestingly, MACE rate was $7.5 \%$ in deferred patients by FFR and $0 \%$ in deferred patients by angiography. 


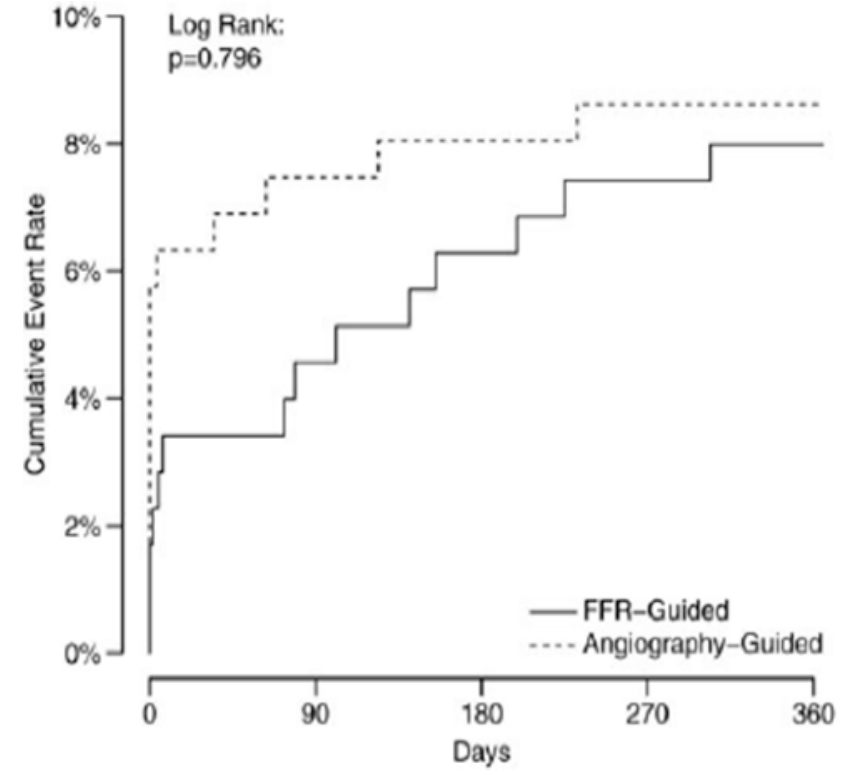

Figure 2 Kaplan-Meier plots of cumulative event rate in patients with acute coronary syndromes enrolled in the FAMOUS-NSTEMI study according to FFR or angioguidance. FFR, fractional flow reserve. (Adapted from Tamita et al). ${ }^{11}$

Taken together as both FAME and FAMOUS-NSTEMI are underpowered, there are no strong data to suggest that FFR guidance improves clinical outcome in ACS. Furthermore, heterogeneity in inclusion criteria, end points and follow-up suggest the need for a larger clinical trial with homogeneous time of assessment, and clear definition of the vessel interrogated by FFR.

\section{POTENTIAL LIMITATIONS OF FFR IN ACS}

A prerequisite of FFR formula calculation is the achievement of so-called 'maximal' hyperaemia due to dilatation of the microcirculation following either intracoronary or intravenous adenosine administration. However, coronary microvascular dysfunction may hamper the response to vasodilators in ACS due to both pre-existing dysfunction or damage to the microcirculation related to ACS. ${ }^{20}$ In this context, there is much evidence to support the notion that in patients with ACS, coronary endothelial dysfunction may pre-exist to the acute coronary event and might even contribute to its pathogenesis. ${ }^{21}$ In particular, reports indicate that abnormal non-endothelium-dependent microvascular dilatation appears to be involved in functional and structural alterations that lead to impaired CFR with ageing, hypertension, diabetes, dyslipidaemia, insulin resistance and chronic inflammatory diseases. ${ }^{22-26}$

Moreover, there is a growing body of evidence to suggest that the presence of endothelial dysfunction is an independent predictor of cardiovascular (CV) events. ${ }^{21}$

Furthermore, in patients with NSTEMI, the coronary microvascular dysfunction distal to the critical stenosis plays an important role in determining the severity of myocardial ischaemia. In particular, a reduction of baseline flow severe enough to impair regional wall motion in remote, normally contracting myocardium subtended by angiographically normal coronary arteries has been observed very early after the infarction. Both coronary microvascular dysfunction and the impairment of regional wall motion are relieved by alpha-blockers, thus suggesting that enhanced sympathetic activation is likely to contribute to these abnormal findings. ${ }^{27}$ Myocardial ischaemia reflexively increases cardiac sympathetic nerve activity by stimulating cardiac ventricular and coronary nerve-ending receptors. ${ }^{28}$

On the other hand, in patients with STEMI, coronary microvascular dysfunction in the territory of a recanalised infarct-related artery is responsible for the MVO, which may lead to lack of perfusion of the myocardium despite the opening of the infarct-related artery. Of note, this phenomenon has been described for STEMI but also for NSTEMI due to embolisation of active plaque material or thrombotic material.

Bax et at assessed CFR and minimal microvascular resistance by combined pressure-flow wires in the culprit vessel of patients with acute MI immediately after reperfusion, then at 1 week and 6 months. CFR increased while minimal microvascular resistance decreased at follow-up in the infarct-related artery. In a recent study, CFR, IMR and FFR were assessed in the culprit lesion at the time of primary PCI, and 6 months later the authors showed that FFR significantly reduced between the acute phase and follow-up reflecting an increase in coronary flow with recovery of the coronary microcirculation. Uniquely, the authors demonstrated a link between IMR and extent of MVO extent on cardiac MRI. ${ }^{7}$ Similar findings were found when flow was assessed by angiography (thrombolysis in myocardial infarction (TIMI) flow grading) as patients with TIMI 2 flow had higher FFR values than those with final TIMI 3 flow in the infarct-related artery. ${ }^{11}$ These studies suggest that FFR assessment of culprit lesion in STEMI may lead to false-negative results during the acute phase.

Nevertheless, prior studies showed a concordance of single-photon emission computed tomography (SPECT) and FFR in the assessment of culprit lesions in MI (both STEMI and NSTEMI) ranging from $71 \%$ to $85 \%$. However, the variable time from ischaemic event to FFR (from 2 days to 6 days), the small sample size of the studies and the inclusion of heterogeneous patient subset (STEMI non-reperfused by primary PCI rarely encountered nowadays, NSTEMI studies by angiography $>24$ hours from chest pain onset not currently recommended by guidelines) do not allow the drawing of a firm conclusion on the accuracy of FFR in the acute setting when the infarct-related artery is interrogated. Finally, non-culprit lesions were not included in such studies, thus data were not provided on the correlation between non-invasive ischaemia and FFR similarly to that available in patients with SA in many studies of FFR validation. 
Although the culprit lesion is often clear in STEMI, not necessitating functional evaluation, the identification of the culprit lesion may be more difficult in NSTEMI where patients often present with MVD. Of note, a recent study by Layland $e t a l^{29}$ failed to show differences in resistive reserve ratio, a measure of the vasodilatory capacity of the microcirculation, in culprit lesions of NSTEMI versus that of SA patients. In this study, IMR tended to be higher (although not statistically different) in culprit vessels of NSTEMI versus that of SA. Again, as in previous studies, IMR was much higher in culprit lesions of STEMI and FFR was also higher as compared with NSTEMI and SA. Importantly, the resistive reserve ratio was negatively correlated with baseline troponin and pre-PCI IMR and tended to be lower (2.46 vs 2.8) in patients with NSTEMI versus those with SA, suggesting that the vasodilatory capacity of the microcirculation may be preserved in some but not all patients with ACS. Finally, the authors assessed these invasive indexes at a mean time of 4 days while currently most patients with ACS and diagnoses of NSTEMI are evaluated within 24 hours from admission-a time frame in which the microcirculation may be more dysfunctional. Thus, the role of FFR in the culprit vessel of NSTEMI needs further studies addressing the effect of time on the assessment of functional indexes of stenosis evaluation.

In non-culprit lesions, although one study reported little change in FFR during the acute phase and follow-up as discussed above, ${ }^{10}$ in the assessment of non-culprit lesions in STEMI other studies have provided conflicting results. Accordingly, lesion severity by angiography has be shown to be exaggerated in non-culprit lesion during the acute phase in ACS in one study, ${ }^{30}$ and hyperaemic microvascular resistance (HMR) was shown to be higher at the time of revascularisation in another study while it was reduced after 6 months both in the infarct and non-infarct-related arteries. ${ }^{10}$ Furthermore, a significant decrease in FFR was shown in a study evaluating non-culprit lesion of STEMI during the acute phase and after a mean of $42 \pm 10$ days $(0.84 \pm 0.08$ vs $0.82 \pm 0.08$, $\mathrm{p}=0.025){ }^{31}$ Finally, discordance between FFR and hyperaemic stenosis resistance (HSR) has been recently suggested by a study comparing FFR and HSR in non-culprit lesions of NSTEMI versus SA. ${ }^{32}$ Discordance was more common in ACS than SA and was related to the variation in microvascular resistance between baseline and hyperaemia (less variation related to more discordancy) (figure 3) and the baseline inflammatory status as assessed by C-reactive protein (CRP) (higher levels related to more discordancy, a finding to be taken into account as CRP may be elevated in as many as $50 \%$ of patients with ACS). As a previous study showed that HSR is a better index of ischaemia than FFR, ${ }^{33}$ these data could suggest that FFR may fail to identify ischaemic lesions in a subset of patients with ACS in non-culprit lesions. Such findings are not surprising as dissociation between FFR and CFR has been previously demonstrated using combined evaluation of microvascular resistances, CFR and FFR in patients with

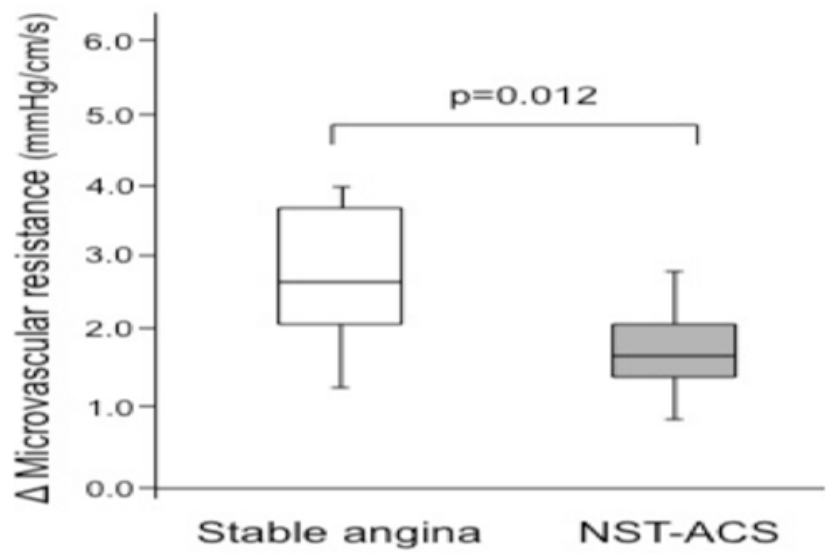

Figure 3 Variation of microvascular resistance in patients with stable angina or non-ST elevation acute coronary syndrome (NST-ACS). (Adapted from Sels et al). ${ }^{18}$

SA and UA. ${ }^{54}$ Such discordance has been suggested to be due to clinically relevant coronary pathophysiology. ${ }^{35}$ In particular, the variability of the microcirculation and flow across the stenosis due to the biologic variability of the stenosis in patients with ACS is therefore to be anticipated. There are several possible mechanisms which may explain this phenomenon in patients with ACS. First, increased release of vasoconstrictors has been demonstrated previously in ACS. Accordingly, Heusch ${ }^{36}$ showed that adenosine is not able to eliminate $\alpha$-adrenergic and endothelin-mediated coronary vasoconstriction, and possibly vasoconstriction induced by other powerful mediators such as angiotensin, serotonin and thromboxane A2. Second, non-culprit plaque in ACS may have vulnerable features and may also be associated with plaque rupture and distal embolisation that hamper the response of the microvessels to vasodilators. ${ }^{37}$ Moreover, a study by Tomai et alfound that in the presence of elevated CRP, patients with NSTEMI showed a reduced response to substance $\mathrm{P}^{38}$ This elevated level of CRP suggests the presence of endothelial dysfunction. Furthermore, CRP levels have been associated with levels of vasoconstrictors and structural alterations of the microcirculation in cases of systemic inflammatory activation. ${ }^{19}$ Again, the effects of elevation of left ventricular (LV) end-diastolic pressure should not be underestimated, and may cause a significant underestimation of lesion severity by FFR. This is particularly prominent in the acute phase. ${ }^{39}$ Finally, in the case of plaque rupture, the severity of stenosis of the acute coronary lesion may fluctuate due to thrombus incorporation in the lesion or vessel remodelling.

In order to try and overcome the resistance to the use of vasodilators in patients with ACS, much attention is paid to the route and dosage of hyperaemic agents such as adenosine. ${ }^{1}$ Under such circumstances, the usual dosing of adenosine may be insufficient to achieve maximal hyperaemia. To overcome this problem, some studies have suggested that, by increasing the adenosine dosage (up to $600 \mathrm{mcg}$ ), FFR may be of benefit. ${ }^{40}{ }^{41}$ However, these protocols have been employed only in patients 
Table 1 All the functional parameters tested in patients with acute coronary syndrome

\begin{tabular}{lllll}
\hline Parameter & Definition & Formula & Abnormal value & Outcome \\
\hline FFR & $\begin{array}{l}\text { Ratio of mean distal coronary } \\
\text { pressure to mean aortic } \\
\text { pressure during maximal } \\
\text { hyperaemia }\end{array}$ & $\begin{array}{l}\text { Pdistal/Paorta } \\
\text { during hyperaemia }\end{array}$ & $\leq 0.80$ & $\begin{array}{l}\text { Coronary stenosis functional } \\
\text { severity }\end{array}$ \\
iFR & $\begin{array}{l}\text { Measurement of } \\
\text { intracoronary pressure } \\
\text { during the diastolic 'wave- }\end{array}$ & $\begin{array}{l}\text { Pdistal/Paorta during 'wave } \\
\text { free period' }\end{array}$ & $<0.90$ & $\begin{array}{l}\text { Coronary stenosis functional } \\
\text { severity }\end{array}$
\end{tabular}

during the diastolic 'wave-

free' period

\begin{tabular}{|c|c|c|c|c|}
\hline CFR & $\begin{array}{l}\text { Ratio of mean distal coronary } \\
\text { peak flow velocity during } \\
\text { maximal hyperaemia to } \\
\text { mean peak flow velocity at } \\
\text { rest }\end{array}$ & $\begin{array}{l}\text { Velocity during hyperaemia/ } \\
\text { velocity during basal } \\
\text { conditions }\end{array}$ & $<2^{\star}$ & $\begin{array}{l}\text { Coronary resistance } \\
\text { Of note, a reduced CFR can } \\
\text { be caused by a significant } \\
\text { epicardial stenosis, by } \\
\text { coronary microvascular } \\
\text { dysfunction or by both }\end{array}$ \\
\hline IMR & $\begin{array}{l}\text { Product of distal coronary } \\
\text { pressure by the mean } \\
\text { transit time during maximal } \\
\text { hyperaemia }\end{array}$ & $\begin{array}{l}\text { Distal pression* mean transit } \\
\text { time during hyperaemia }\end{array}$ & $<32-35 U$ & Microvascular resistance \\
\hline
\end{tabular}

\begin{abstract}
${ }^{*}$ As research on IMR and HMR is scarce, no general cut-off values have yet been proposed. In clinical studies, different cut-off values for IMR between 32 and $35 \mathrm{U}$ are used and for HMR a cut-off value of $3.25 \mathrm{~mm} \mathrm{Hg} / \mathrm{cm} \cdot \mathrm{s}$ depending on the median value in the respective study group and/or ROC curves is used. For CFR a cut-off value of 2.0 is generally accepted.

CFR, coronary flow reserve; FFR, fractional flow reserve; HMR, hyperaemic microvascular resistance; HSR, hyperaemic stenosis resistance; iFR, instantaneous wave-free ratio; IMR, index of microvascular resistance; Paorta, proximal perfusion pressure; Pdistal, distal coronary pressure; ROC, receiver operating characteristic.
\end{abstract}

with stable $\mathrm{CAD}$, and therefore cannot be recommended in ACS. Of note, if escalation of adenosine dosing may increase hyperaemia, it carries a greater risk, particularly in the unstable setting of further antagonism of unstable haemodynamics. In such scenarios, high-dose intravenous administration of adenosine may lead to false-positive FFR results in addition to an acute and undesirable deterioration in haemodynamics. ${ }^{42}$ When making an assessment in the acute setting, the potential for such undesirable deterioration should always be considered, especially when there is a large myocardial mass and depressed LV function is involved.

\section{OTHER FUNCTIONAL INDEXES TO ASSESS CORONARY STENOSIS IN ACS}

Based on the considerations stated above, the search for the best index of functional stenosis severity evaluation in ACS is still ongoing. Table 1 reports all the functional parameters tested in patients with ACS. Two approaches need to be evaluated in ACS: the use of pressure-flow wires that allow the combined assessment of stenosis and microvascular resistance alongside FFR, and newer pressure-only adenosine-free indexes such as iFR. However, the impact of microvascular dysfunction and plaque activity on such indexes should be a matter of future studies. Of note, iFR has recently been suggested to have better correlation with CFR than FFR. ${ }^{43}$ Interestingly, the closer relationship between iFR and CFR is lost when iFR is measured during adenosine administration, suggesting that the hyperaemic response itself is the most likely cause of conflicts between pressure indexes and CFR.

\section{HSR index}

HSR is calculated by combined pressure-flow assessment of coronary stenosis severity. ${ }^{44}$ It is calculated by dividing the trans-stenotic pressure gradient by mean flow under 
hyperaemic conditions. HSR is less dependent than FFR on microvascular function, and it has been shown to be superior to FFR for ischaemia detection by myocardial perfusion imaging in patients with SA. ${ }^{44}$ Pressure-flow wires can be used to calculate the HMR, defined as the ratio of mean distal pressure divided by mean distal flow under hyperaemic conditions. Myocardial resistance has an important effect on any physiological index such as FFR. This is because the relationship between FFR and HSR is modulated by the magnitude of HMR, such that for any given level of epicardial disease, equivalent FFR severity increases with increasing resistance. ${ }^{44}$ As HSR is less susceptible to resistance changes than FFR, it may be superior in detecting ischaemia-producing lesions in acute patients. To date, a direct comparison between FFR and HSR in ACS patient populations remains lacking.

\section{iFR for evaluation of stenosis severity in stable patients and patients with ACS}

Despite the strong evidence base for FFR, the current use of FFR in patients with ACS remains rather low worldwide. Reasons for this are multifactorial, including: lack of sufficient device reimbursement, increased procedural time and costs for adenosine administration, contraindication in patients with asthma and chronic obstructive pulmonary disease, and residual reliance on visual assessment of coronary angiography. An alternative adenosine-free index of coronary stenosis, iFR, was recently introduced in patients with stable coronary disease to assess the stenosis severity. ${ }^{45-47}$ In particular, iFR, a pressure-only index, has some characteristic features that make it attractive for physiological assessment during ACS. iFR is calculated from the mean distal to aortic mean pressure ratio over a period of diastole which is absent from active contraction of relaxation (ie, the wave-free period). During this phase, resistance is at its lowest and most stable over the cardiac cycle. This provides the optimum window for physiological measurements to be made. iFR has been evaluated in large registries in real life, showing good agreement with FFR and at least similar discriminative power for ischaemia detection when compared with FFR. ${ }^{45-47}$ Of note, iFR, when compared with FFR, shows stronger correlation and better agreement with CFR, suggesting that iFR may be better suited for stenosis severity evaluation in cases of dissociation between FFR and CFR, a potential situation in $\mathrm{ACS}^{43}$ (figure 4). Interestingly, iFR/FFR correlation shows no difference between patients with ACS and stable patients in a recent study. ${ }^{48}$ The Forecast Trial was the first study that evaluated the iFR for the assessment of non-culprit vessels in patients with ACS. ${ }^{39}$ A total of 123 stenoses were evaluated with both FFR and iFR. Classification match of iFR in ACS was not inferior to stable CAD (79.5\% in ACS and $84.4 \%$ in CAD; $p=0.497$ ) (figure 5). Accordingly, no difference was observed in iFR/FFR correlation between ACS and stable CAD ( $\mathrm{r}=0.66$ in ACS vs $r=0.69$ in $\mathrm{CAD})$. Overall, a significant correlation was found between iFR and FFR ( $r=0.68 ; p=0.001)$ with a good diagnostic efficiency at receiver operating characteristic

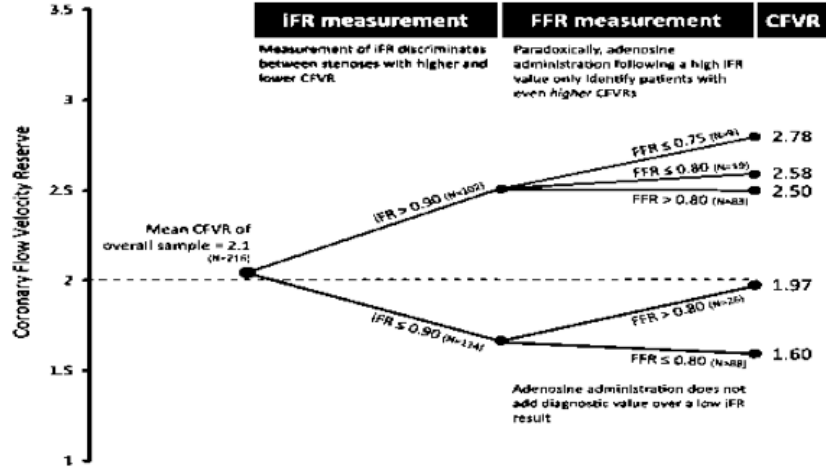

Figure 4 Agreement between CFR, FFR and iFR. When CFR is below 2, $23 \%$ of patients may have normal FFR $(>0.80)$ and the agreement between CFR and iFR is much better than that of CFR and FFR. Such scenario may be common in acute coronary syndromes with low CFR. CFR, coronary flow reserve; CFVR, coronary flow velocity reserve; FFR, fractional flow reserve; iFR, instantaneous wave-free ratio. (Adapted from Tomai et al). ${ }^{38}$

analysis (area under the curve: 0.87). In addition, neither the localisation of the stenosis within the coronary tree $(p=0.147)$ nor the time interval from the acute event $(\mathrm{p}=0.550)$ significantly influenced the concordance of iFR with FFR. Therefore, the iFR, during the wave-free period and without hyperaemic stimulation, is feasible in patients with ACS. Indeed, it is a promising diagnostic technique for the evaluation of non-culprit intermediate stenoses in patients with ACS, and its classification match to FFR in such a clinical context is not inferior compared with stable CAD. ${ }^{48}$ However, it has to be stated, although there is a good classification match between the two indexes, it does not achieve complete agreement. In particular, as a resting measure, iFR is more consistent across microvascular and non-microvascular dysfunction

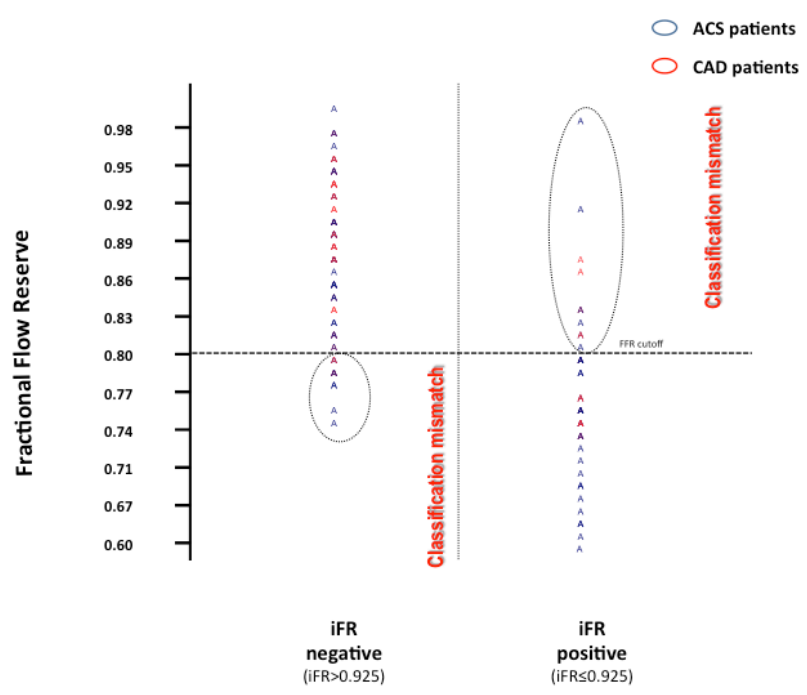

Figure 5 Assessment of classification mismatch between iFR and FFR. ACS, acute coronary syndrome; CAD, coronary artery disease; FFR, fractional flow reserve; iFR, instantaneous wave-free ratio. (Adapted from EchavarríaPinto et al). ${ }^{42}$ 
states. Importantly, ischaemic FFR values $(\leq 0.75)$ may be generated by high hyperaemic flow rates and higher-than-average CFR. Specifically, the generation of large hyperaemic gradients in stenoses with normal iFR values ( $>0.9$ ) identifies a particular subgroup of patients with high CFR. These lesions demonstrate, on average, magnitudes of hyperaemic flow velocities equal to what is observed in stenoses with FFR $>0.80$, both significantly higher than flow velocities seen in the overall population of FFR-significant lesions $(\leq 0.75)$. Conversely, an increase in microvascular resistance due to microvascular disease or submaximal hyperaemia will be reflected in an increase in distal coronary pressure, and thus in an increase in FFR, while the hampered flow through the stenosis will result in a decrease in CFR, and vice versa. In this context, the IFR could be helpful to avoid the false-negative FFR values in ACS, as slightly more patients are 'positive' with iFR with microcirculatory disease. This phenomenon depends on the population under study and is more pronounced in microvascular dysfunction state such as ACS. Thus, taken together, these data are supportive of the potential future role of iFR as a tool to guide decision making in ACS. While the current data provide an interesting background to the relationship between non-hyperaemic and hyperaemic indices, prior to the results of large randomised clinical trials, it is not possible to know whether iFR is superior to FFR in the evaluation of coronary stenoses in patient with ACS. Two large prospective randomised clinical trials, FLAIR (NCT02053038) and SWEDEHEART (NCT02166736), will further address the use of iFR and FFR in the diagnostic evaluation of stenosis severity for the assessment of intermediate stable coronary stenoses, and non-culprit stenoses in ACS. Patients will be recruited from standard clinical work flows, and it is anticipated that up to $50 \%$ of patients evaluated will be in the ACS arm. Moreover, the FLAIR study will look at MACE, and cost-effectiveness at 1 year using established iFR and FFR dichotomous cut-off points.

\section{CLINICAL IMPLICATION OF DEFERRING CORONARY STENOSIS TREATMENT IN ACS VERSUS SA}

In the DEFER trial, ${ }^{3}$ deferral of patients with stable CAD and FFR $<0.75$ was associated with a very low event rate (around 3\%) after 5 years. Based on this study and others, FFR has attained a class IA level of recommendation in the latest ESC guidelines on myocardial revascularisation. ${ }^{2}$ Most of the evidence is gained from patients with stable CAD with few little data from patients at higher risk of events associated with deferral of treatment in ACS. Notably, a recent study has suggested that FFR has a continuous and independent relationship with subsequent outcomes, suggesting that borderline FFR value may still benefit from revascularisation, ${ }^{49}$ while another study has suggested accordingly that the risk of deferred lesion intervention may be modulated by clinical factors as well as FFR values. ${ }^{50}$ Lopez-Palop et $a l^{51}$ showed a similar event rate in non-culprit lesion assessed by FFR (with a 0.75 cut-off) when comparing treated versus deferred lesions $(7.4 \%$ vs $7.7 \%)$, an event rate higher than that observed after 1 year in the DEFER study. Similarly, Fischer $e t a \tilde{l}^{2}$ showed that, by using a 0.75 cut-off, deferred lesions in non-culprit vessels of ACS versus those with no ACS had a trend for higher event rate $(28 \%$ vs $17 \%)$. Finally, in an all-comers ACS population (NSTEMI and STEMI $>24$ hours) deferring treatment with a cut-off of 0.75 was associated with an event rate related to the deferred lesion of $7.5 \% .^{53}$ These studies suggest that the risk associated with deferring lesions in non-culprit territories of ACS is higher than that expected for deferred SA lesions based on FFR.

However, the natural history of the coronary lesions of patients with ACS is different from its stable counterpart. Moreover, compared with patients with single vessel disease, individuals with ACS and MVD presented a poorer outcome. It is still unclear if this phenomenon is attributable to an increased disease burden and activity or because ischaemia-producing lesions in other territories are left untreated. ${ }^{54}$ Some studies have suggested that patients with MI and MVD have a considerable proportion of unstable and vulnerable lesions in addition to the identified culprit artery. ${ }^{55}$ Thus, the importance of FFR assessment could be less pronounced, as the likelihood of vulnerable plaque rupture is not strictly related to FFR, but to the underlying activity of the disease. Indeed, the plaque instability independent of the stenosis severity itself may be driver of the events in the ACS. It means that FFR may be inaccurate in predicting the risk of future events when their values are negative and when they are positive.

Finally, a recent study ${ }^{6}$ on a mixed population of patients with SA and ACS showed that in borderline values of FFR (between 0.8 and 0.85 ) the risk of events is particularly high (HR 1.63, 95\% CI 1.14 to 2.33). The data presented are not surprising as some deferred lesion, especially those in the borderline zone, may represent false-negative results of FFR due to high HMR and low CFR. Of note, HMR is associated with ischaemia independently of FFR ${ }^{56}$ while an abnormal CFR with a normal FFR is associated with a worse prognosis after 10 years of follow-up in the case of stable patients. ${ }^{27}$ These observations are probably related to the strong prognostic role of coronary microvascular dysfunction in addition to that conferred by stenosis severity, as clearly suggested by studies using positron emission tomography. ${ }^{5758}$

\section{FUTURE PERSPECTIVES AND CONCLUSIONS}

Patients with ACS represent a different subset of CAD characterised often by microvascular dysfunction and transient coronary vasoconstriction that may affect FFR results. This notion should be taken into account as deferring lesion with high FFR values especially in the borderline zone which may lead to recurrent events. Whether this is due to undertreatment of severe stenosis 
or to worse microvascular function has not be tested to date. However, other indexes of functional stenosis evaluation with better correlation with microvascular function may possibly be superior to FFR. At present, physicians should remain vigilant that borderline FFR values in patients with ACS may be underestimates of true severity stenosis.

Contributors All the authors: 1) have provided substantial contributions to the conception or design of the work, or the acquisition, analysis or interpretation of data; 2) drafted the work and revised it critically for important intellectual content; 3) approved the version published.

\section{Competing interests None declared.}

Open Access This is an Open Access article distributed in accordance with the Creative Commons Attribution Non Commercial (CC BY-NC 4.0) license, which permits others to distribute, remix, adapt, build upon this work non-commercially, and license their derivative works on different terms, provided the original work is properly cited and the use is non-commercial. See: http://creativecommons.org/ licenses/by-nc/4.0/

(C) Article author(s) (or their employer(s) unless otherwise stated in the text of the article) 2017. All rights reserved. No commercial use is permitted unless otherwise expressly granted.

\section{REFERENCES}

1. Pijls NH, Sels JW. Functional measurement of coronary Stenosis. J Am Coll Cardiol 2012;59:1045-57.

2. Kolh P, Windecker S. ESC/EACTS myocardial revascularization guidelines 2014. Eur Heart J 2014;35:3235-6.

3. Pijls NH, van Schaardenburgh P, Manoharan G, et al. Percutaneous coronary intervention of functionally nonsignificant Stenosis: 5-year follow-up of the DEFER Study. J Am Coll Cardiol 2007;49:2105-11.

4. Tonino PA, De Bruyne B, Pijls NH, et al. Fractional flow reserve versus angiography for guiding percutaneous coronary intervention. N Engl J Med 2009;360:213-24.

5. Echavarría-Pinto M, van de Hoef TP, Serruys PW, et al. Facing the complexity of ischaemic heart disease with intracoronary pressure and flow measurements: beyond fractional flow reserve interrogation of the coronary circulation. Curr Opin Cardiol 2014;29:564-70.

6. Depta JP, Patel JS, Novak E, et al. Outcomes of coronary stenoses deferred revascularization for borderline versus nonborderline fractional flow reserve values. Am J Cardiol 2014;113:1788-93.

7. Cuculi F, De Maria GL, Meier P, et al. Impact of microvascular obstruction on the assessment of coronary flow reserve, index of microcirculatory resistance, and fractional flow reserve after ST-segment elevation myocardial infarction. J Am Coll Cardiol 2014;64:1894-904.

8. McCormick LM, Hoole SP, Brown AJ, et al. A contemporary reevaluation of culprit lesion severity in patients presenting with STEMI. Acute Card Care 2012;14:111-6.

9. Bax M, de Winter RJ, Koch KT, et al. Time course of microvascular resistance of the infarct and noninfarct coronary artery following an anterior wall acute myocardial infarction. Am J Cardiol 2006;97:1131-6.

10. Hoebers LP, Vis MM, Claessen BE, et al. The impact of multivesse disease with and without a co-existing chronic total occlusion on short- and long-term mortality in ST-elevation myocardial infarction patients with and without cardiogenic shock. Eur J Heart Fail 2013;15:425-32

11. Tamita K, Akasaka T, Takagi T, et al. Effects of microvascular dysfunction on myocardial fractional flow reserve after percutaneous coronary intervention in patients with acute myocardial infarction. Catheter Cardiovasc Interv 2002;57:452-9.

12. De Bruyne B, Pijls NH, Bartunek J, et al. Fractional flow reserve in patients with prior myocardial infarction. Circulation 2001;104:157-62.

13. Samady H, Lepper W, Powers ER, et al. Fractional flow reserve of infarct-related arteries identifies reversible defects on noninvasive myocardial perfusion imaging early after myocardial infarction. J Am Coll Cardiol 2006;47:2187-93.

14. Layland J, Rauhalammi S, Watkins S, et al. Assessment of Fractional flow Reserve in Patients with recent Non-ST-Segment-Elevation myocardial infarction: comparative study with 3-T stress Perfusion cardiac magnetic resonance Imaging. Circ Cardiovasc Interv 2015;8:e002207.
15. Ntalianis A, Sels JW, Davidavicius G, et al. Fractional flow reserve for the assessment of nonculprit coronary artery stenoses in patients with acute myocardial infarction. JACC Cardiovasc Interv 2010;3:1274-81.

16. Indolfi C, Piscione F, Ceravolo R, et al. Limb vasoconstriction after successful angioplasty of the left anterior descending coronary artery. Circulation 1995;92:2109-12.

17. Engstrøm T, Kelbæk H, Helqvist S, et al. Complete revascularisation versus treatment of the culprit lesion only in patients with STsegment elevation myocardial infarction and multivessel disease (DANAMI-3-PRIMULTI): an open-label, randomised controlled trial. Lancet 2015;386:665-71.

18. Sels JW, Tonino PA, Siebert U, et al. Fractional flow reserve in unstable angina and non-ST-segment elevation myocardial infarction experience from the FAME (Fractional flow reserve versus angiography for Multivessel evaluation) study. JACC Cardiovasc Interv 2011;4:1183-9.

19. Layland J, Oldroyd KG, Curzen N, et al. Fractional flow reserve vs. angiography in guiding management to optimize outcomes in non-ST-segment elevation myocardial infarction: the british Heart Foundation FAMOUS-NSTEMI randomized trial. Eur Heart J 2015;36:100-11.

20. Camici PG, Crea F. Coronary microvascular dysfunction. N Engl J Med 2007;356:830-40.

21. Lerman A, Zeiher AM. Endothelial function: cardiac events. Circulation 2005;111:363-8.

22. Panza JA, Quyyumi AA, Brush JE, et al. Abnormal endotheliumdependent vascular relaxation in patients with essential hypertension. N Engl J Med 1990;323:22-7.

23. Mäkimattila S, Virkamäki A, Groop PH, et al. Chronic hyperglycemia impairs endothelial function and insulin sensitivity via different mechanisms in insulin-dependent diabetes mellitus. Circulation 1996;94:1276-82.

24. Casino PR, Kilcoyne CM, Quyyumi AA, et al. The role of nitric oxide in endothelium-dependent vasodilation of hypercholesterolemic patients. Circulation 1993;88:2541-7.

25. Celik T, Turhan $\mathrm{H}$, Kursaklioglu $\mathrm{H}$, et al. Impact of metabolic syndrome on myocardial perfusion grade after primary percutaneous coronary intervention in patients with acute ST elevation myocardial infarction. Coron Artery Dis 2006;17:339-43.

26. Schindler TH, Nitzsche EU, Olschewski M, et al. Chronic inflammation and impaired coronary vasoreactivity in patients with coronary risk factors. Circulation 2004;110:1069-75.

27. Gregorini L, Marco J, Kozàkovà M, et al. Alpha-adrenergic blockade improves recovery of myocardial perfusion and function after coronary stenting in patients with acute myocardial infarction Circulation 1999;99:482-90.

28. Heusch G, Deussen A, Thämer V. Cardiac sympathetic nerve activity and progressive vasoconstriction distal to coronary stenoses: feed-back aggravation of myocardial ischemia. J Auton Nerv Syst 1985;13:311-26.

29. Layland J, Carrick D, McEntegart M, et al. Vasodilatory capacity of the coronary microcirculation is preserved in selected patients with non-ST-segment-elevation myocardial infarction. Circ Cardiovasc Interv 2013;6:231-6.

30. Hanratty CG, Koyama $\mathrm{Y}$, Rasmussen $\mathrm{HH}$, et al. Exaggeration of nonculprit Stenosis severity during acute myocardial infarction: implications for immediate multivessel revascularization. J Am Coll Cardiol 2002;40:911-6.

31. Wood DA, Poulter R, Boone R, et al. Stability of Non Culprit Vessel Fractional flow Reserve in Patients with St-Segment Elevation myocardial infarction. Can J Cardiol 2013;29:S291-2.

32. Niccoli G, Falcioni E, Cosentino N, et al. Impact of accuracy of fractional flow reserve to reduction of microvascular resistance after intracoronary adenosine in patients with angina pectoris or non-ST-segment elevation myocardial infarction. Am J Cardiol 2014:113:1461-7.

33. Meuwissen M, Siebes M, Chamuleau SA, et al. Hyperemic Stenosis resistance index for evaluation of functional coronary lesion severity. Circulation 2002;106:441-6.

34. van de Hoef TP, van Lavieren MA, Damman P, et al. Physiological basis and long-term clinical outcome of discordance between fractional flow reserve and coronary flow velocity reserve in coronary stenoses of intermediate severity. Circ Cardiovasc Interv 2014;7:301-11.

35. Johnson NP, Kirkeeide RL, Gould KL. Is discordance of coronary flow reserve and fractional flow reserve due to methodology or clinically relevant coronary pathophysiology? JACC Cardiovasc Imaging 2012;5:193-202.

36. Heusch $\mathrm{G}$. Adenosine and maximum coronary vasodilation in humans: myth and misconceptions in the assessment of coronary reserve. Basic Res Cardiol 2010;105:1-5. 
37. Buffon A, Biasucci LM, Liuzzo G, et al. Widespread coronary inflammation in unstable angina. N Engl J Med 2002;347:5-12.

38. Tomai F, Ribichini F, Ghini AS, et al. Elevated C-reactive protein levels and coronary microvascular dysfunction in patients with coronary artery disease. Eur Heart $J$ 2005;26:2099-105.

39. Van Herck PL, Carlier SG, Claeys MJ, et al. Coronary microvascular dysfunction after myocardial infarction: increased coronary zero flow pressure both in the infarcted and in the remote myocardium is mainly related to left ventricular filling pressure. Heart 2007; $93: 1231-7$

40. Leone AM, Porto I, De Caterina AR, et al. Maximal hyperemia in the assessment of fractional flow reserve: intracoronary adenosine versus intracoronary sodium nitroprusside versus intravenous adenosine: the NASCI (Nitroprussiato versus Adenosina nelle Stenosi Coronariche Intermedie) study. JACC Cardiovasc Interv 2012;5:402-8.

41. Adjedj J, Toth GG, Johnson NP, et al. Intracoronary Adenosine: dose-response relationship with hyperemia. JACC Cardiovasc Interv 2015;8:1422-30.

42. Echavarría-Pinto M, Gonzalo N, Ibañez B, et al. Low coronary microcirculatory resistance associated with profound hypotension during intravenous adenosine infusion: implications for the functional assessment of coronary stenoses. Circ Cardiovasc Interv 2014;7:35-42.

43. Petraco R, van de Hoef TP, Nijjer S, et al. Baseline instantaneous wave-free ratio as a pressure-only estimation of underlying coronary flow reserve: results of the JUSTIFY-CFR study (Joined coronary pressure and flow analysis to determine diagnostic characteristics of basal and hyperemic indices of functional lesion Severity-Coronary flow Reserve). Circ Cardiovasc Interv 2014;7:492-502.

44. van de Hoef TP, Nolte F, Echavarrla-Pinto M, et al. Impact of hyperaemic microvascular resistance on fractional flow reserve measurements in patients with stable coronary artery disease: insights from combined Stenosis and microvascular resistance assessment. Heart 2014;100:951-9.

45. Sen S, Escaned J, Malik IS, et al. Development and validation of a new adenosine-independent index of Stenosis severity from coronary wave-intensity analysis: results of the ADVISE (ADenosine vasodilator Independent Stenosis evaluation) study. J Am Coll Cardiol 2012;59:1392-402.

46. Sen S, Asrress KN, Nijjer S, et al. Diagnostic classification of the instantaneous wave-free ratio is equivalent to fractional flow reserve and is not improved with adenosine administration. results of CLARIFY (Classification accuracy of Pressure-Only Ratios against Indices using flow study). J Am Coll Cardiol 2013;61:1409-20.
47. Nijjer SS, Sen S, Petraco R, et al. Advances in coronary physiology. Circ J 2015;79:1172-84.

48. Indolfi C, Mongiardo A, Spaccarotella C, et al. The instantaneous wave-free ratio (iFR) for evaluation of non-culprit lesions in patients with acute coronary syndrome and multivessel disease. Int $\mathrm{J}$ Cardiol 2015;178:46-54.

49. Johnson NP, Tóth GG, Lai D, et al. Prognostic value of fractional flow reserve: linking physiologic severity to clinical outcomes. J Am Coll Cardiol 2014;64:1641-54.

50. Depta JP, Patel JS, Novak E, et al. Risk model for estimating the 1-year risk of deferred lesion intervention following deferred revascularization after fractional flow reserve assessment. Eur Heart J 2015;36:509-15.

51. Lopez-Palop R, Carrillo P, Torres F, et al. Results of fractional flow reserve measurement to evaluate nonculprit coronary artery stenoses in patients with acute coronary syndrome. Rev Esp Cardiol 2012;65:164-70.

52. Fischer JJ, Wang XQ, Samady $\mathrm{H}$, et al. Outcome of patients with acute coronary syndromes and moderate coronary lesions undergoing deferral of revascularization based on fractional flow reserve assessment. Catheter Cardiovasc Interv 2006;68:544-8.

53. Potvin JM, Rodés-Cabau J, Bertrand OF, et al. Usefulness of fractional flow reserve measurements to defer revascularization in patients with stable or unstable angina pectoris, non-ST-elevation and ST-elevation acute myocardial infarction, or atypical chest pain. Am J Cardiol 2006;98:289-97.

54. Politi L, Sgura F, Rossi R, et al. A randomised trial of target-vessel versus multi-vessel revascularisation in ST-elevation myocardia infarction: major adverse cardiac events during long-term follow-up. Heart 2010;96:662-7.

55. Goldstein JA, Demetriou D, Grines CL, et al. Multiple complex coronary plaques in patients with acute myocardial infarction. $N$ Engl $J$ Med 2000;343:915-22.

56. Nolte F, van de Hoef TP, Meuwissen M, et al. Increased hyperaemic coronary microvascular resistance adds to the presence of myocardial ischaemia. Eurolntervention 2014:9:1423-31.

57. Gould KL, Johnson NP, Bateman TM, et al. Anatomic versus physiologic assessment of coronary artery disease. role of coronary flow reserve, fractional flow reserve, and positron emission tomography imaging in revascularization decision-making. J Am Coll Cardiol 2013:62:1639-53.

58. Dorbala S, Di Carli MF. Cardiac PET perfusion: prognosis, risk stratification, and clinical management. Semin Nucl Med 2014;44:344-57. 